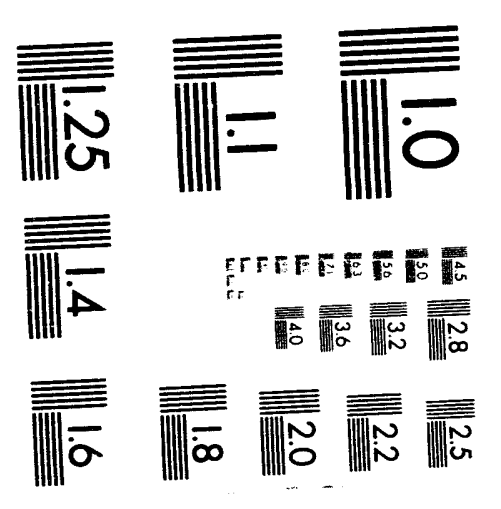



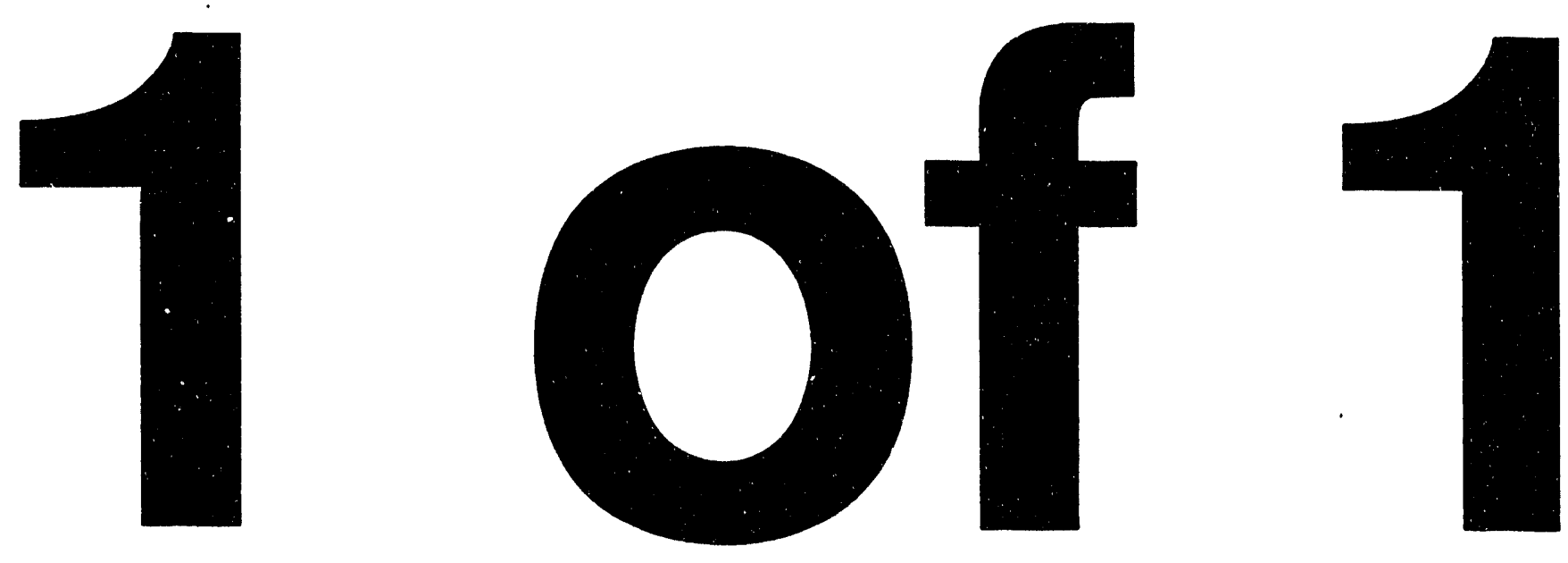


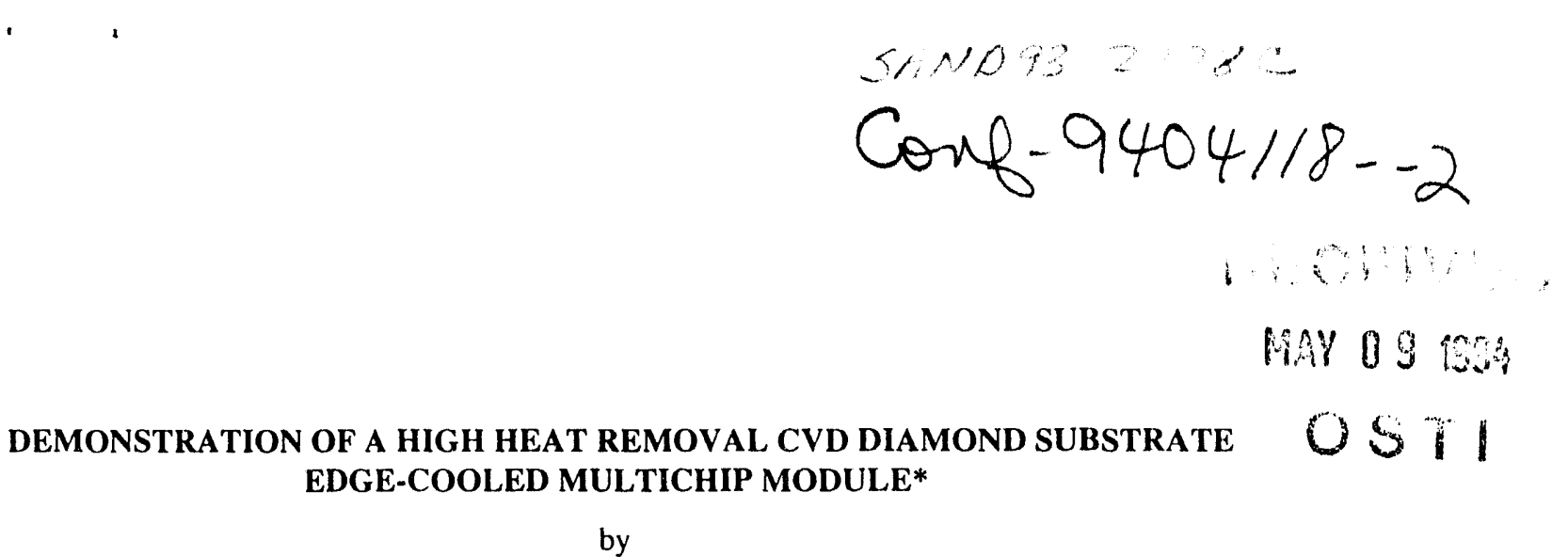

\author{
David W. Peterson \\ Sandia National Laboratories \\ Albuquerque, NM 87185-1082 \\ P: 505-844-6009 \\ F: 505-844-2991 \\ James N. Sweet, David D. Andaleon, Ronald F. Renzi \\ Sandia National Laboratories \\ Albuquerque, NM 87185 \\ \& \\ Donald R. Johnson \\ Allied Signal Corp. \\ Kansas City, MO 64131
}

\begin{abstract}
A single substrate intended for a 3-dimensional (3D) edge-cooled multichip module (MCM) has been built and thermally tested. The substrate, with dimensions 1.9 in. by 2 in., is mounted in a fluid cooled block at one end. To test this cooling architecture and verify the accuracy of thermal models, we constructed thermal test modules using alumina $\left(\mathrm{Al}_{2} \mathrm{O}_{3}\right)$, aluminum nitride $(\mathrm{AlN})$, and $\mathrm{CVD}$ diamond substrate materials. Each module was populated with an array of 16 Sandia ATCO3 test chips with resistive heaters and temperature sensing diode thermometers.
\end{abstract}

Comparative measurements of the 3 substrates were made in which the top row of 4 die were heated at 5 Weach for a total of $20 \mathrm{~W}$. The maximum temperature differences between the heated die and the interface with the cold chuck, $\Delta T_{j s}$ were 24,126 , and $265^{\circ} \mathrm{C}$ for diamond, AlN and alumina, respectively. Measurements on the diamond thermal test module, uniformly heated at a total power of $40 \mathrm{~W}$, gave a measured junction-to-sink temperature of $\Delta T_{j,} \approx$ $18^{\circ} \mathrm{C}$. This result indicates that the diamond edge-cooled substrate could dissipate a total power $\approx 200 \mathrm{~W}$ for a maximum $\Delta T_{j s}=100^{\circ} \mathrm{C}$. If multiple substrates were mounted in the fluid cooled block, spaced 0.075 in. apart, the volumetric power density would be about $880 \mathrm{~W} / \mathrm{in}$.

\title{
INTRODUCTION
}

The recent introduction of synthetically produced chemical vapor deposited (CVD) diamond substrates has led to the proposal of novel 2D and 3D multichip module designs which capitalize on diamond's high thermal conductivity. The literature reflects a growing interest in applying diamond substrate technology to solving 2D and 3D MCM thermal management problems $[1,2]$. For example, a replacement power electronics module for a flight computer has been designed and fabricated which uses CVD diamond to spread and dissipate large transient power pulses [3 ]. This 2D implementation incorporates high power MOSFETs in die form, and thin film resistor technology for high wattage load resistors and results in a significant reduction in physical size from the PC board version.
3D thermal management has been addressed in a digital computer demonstration program described in [3] and shown conceptually in Fig. 1. One objective of this program was to show how 2D MCMs could be packaged in three dimensions, substantially reducing inter-die signal propagation delay through the use of zaxis optical and electrical conductors. This packaging scheme is possible only if heat from each 2D module can be extracted in some practical way from the edge. The low spreading resistance and high thermal conductivity of CVD diamond make it an obvious candidate for removal of large steady-state heat loads at the edge.

In this work we analyze and provide experimental verification of the heat transport properties of a thermal 
test module fabricated from diamond, AIN, and alumina substrates. This module was designed to fit into the 3D liquid edge-cooled cube shown in Fig. I. In the first section we discuss a simplified ID heat transfer model which predicts the behavior of the test module.

The second section describes the design and construction of the test module, and the third section discusses our experimental results.

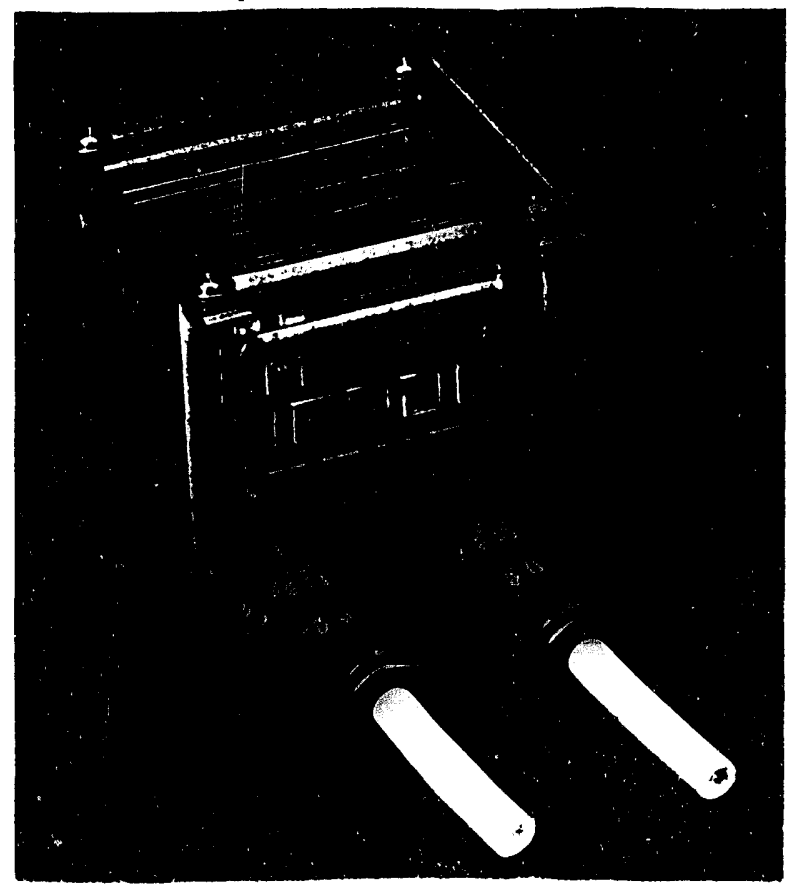

Fig. 1. Conceptual design for a $3 D$ cube using liquid edgecooled diamond substrates. All heat is removed through substrate conduction to the fluid cooled bottom region.

\section{ANALYSIS OF HEAT TRANSFER}

1D heat transfer: Most of the significant heat transfer phenomenon for an edge-cooled MCM substrate can be described with a one-dimensional (ID) heat conduction analysis. Fig. 2 shows a $1 \mathrm{D}$ model for the substrate, with the temperature of the strip varying only in the $x$ direction. The substrate with thermal conductivity $k$ has length $L$, width $w$, and thickness $t$. Since all temperatures are measured relative to the cooled end at $x=0$, we assume that $\left.7\right|_{x=0}=0$. At the far end, $x=L$, we assume an adiabatic boundary with no heat transfer corresponding to the condition, $k \partial T /\left.\partial x\right|_{x=L}=0$. The one dimensional heat conduction equation for this problem with a distributed heat source $q(x) \mathrm{W} / \mathrm{m}^{3}$ is,

$$
\frac{d^{2} T(x)}{d x^{2}}+\frac{q(x)}{k}=0
$$

The solution for a general source $q(x)$ is given by,

$$
T(x)=\frac{\bar{q} L x}{k}-\frac{x}{k} \int_{0}^{1} q\left(x^{\prime}\right) d x^{\prime}+\frac{1}{k} \int_{0}^{1} x^{\prime} q\left(x^{\prime}\right) d x^{\prime}
$$

In Eq.(2), $\bar{q}$ is the average power, given by, $\bar{q}=(1 / L) \int_{0}^{L} q(x) d x$. The first term in Eq. (2) represents the linear temperature profile which would be produced if all of the input power was effectively a line source at $x=L$. The second two terms represent deviations from the linear profile produced by the distributed nature of the source.

To show the dependence of the temperature on the thermal conductivity and geometric properties of the substrate, it is convenient to make the transformation $v=x / L$ in Eq. (2). This transformation yields the relation for the temperature in terms of the dimensionless length $v$,

$$
T(v)=\left(\frac{L}{k w t}\right) P_{T}\left\{v-\int_{0}^{v}\left(v-v^{\prime}\right) q_{r}\left(v^{\prime}\right) d v^{\prime}\right\}
$$

In Eq. (3), $P_{T}$ is the total power into the substrate and $q_{r}(v)$ is the relative power density defined by $q_{r}(v)=\left(L w t / P_{T}\right) q(v) \cdot q_{r}(v)$ has the property that $\int_{0}^{1} q_{r}(v) d v=1$. The first factor in Eq. (3), $L(k w t)$ is the thermal resistance of the substrate to uniform or axial heat flow. We define this to be the characteristic thermal impedance,

$$
\theta_{0}=L /(k w t)
$$

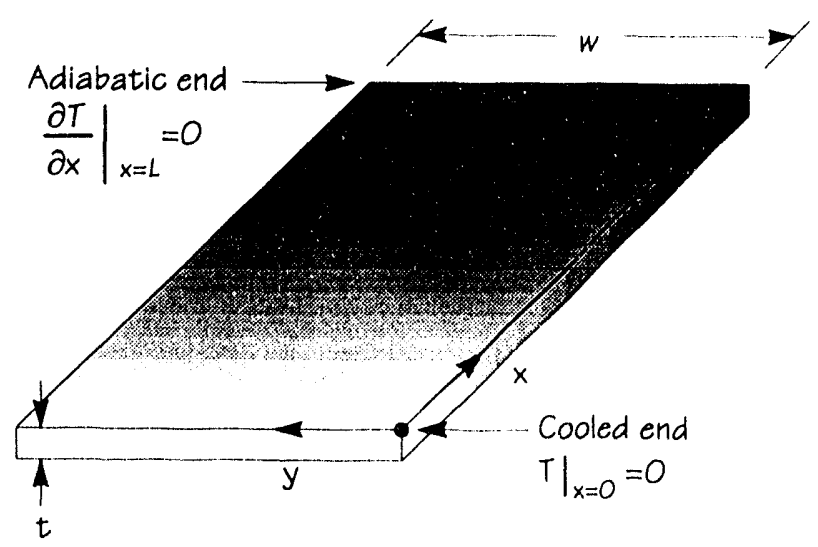

Fig. 2. Model used for one-dimensional heat conduction analysis of edge-cooled substrate, where $t=$ thickness, $w=$ width, and $L=$ length.

We now consider the case in which the power density is constant in a strip parallel to the $y^{\prime}$ axis, $x_{1} \leq x_{1} \leq x_{2}$. corresponding to $v_{1} \leq v \leq v_{2}$. In this case, $q_{1}\left(v^{\prime}\right)=1 / \Delta \cdot$ for $v_{1} \leq v \leq v_{2}$, and () outside this region, where $A v=1,1$, . The 
solution, $T_{s}\left(v \mid v_{1}, v_{2}\right)$ for the temperature produced by the heated strip in $v_{1} \leq v \leq v_{2}$ can be written in the form,

$$
\begin{array}{ll}
T_{s}\left(v \mid v_{1}, v_{2}\right)=\theta_{0} P_{T} f\left(v \mid v_{1}, v_{2}\right) & 0 \leq v \leq v_{1} \\
\text { where } & \\
f=v & v_{1} \leq v \leq v_{2} \\
f=\left[v-\frac{1}{\Delta v}\left(\frac{v^{2}}{2}+\frac{v_{1}^{2}}{2}-v_{1} v\right)\right] & v_{2} \leq v \leq 1
\end{array}
$$

Eq. (5) shows that the temperature varies linearly with position for $v$ to the left of the heated region and quadratically within the heated region. Between the right boundary of the heated region and the adiabatic right boundary of the substrate, the temperature is constant. In a case with several heated strips, the temperature is given by,

$$
T(v)=\theta_{0} P_{T} \sum_{i} f\left(v \mid v_{1 i}, v_{2 i}\right) p_{r i}
$$

In Eq.(6), the sum is over the heated strips and $p_{r i}$ is the fraction of the total power in the ith strip. With this definition for the $p_{r i}$, they obey the condition,

$\sum_{i} p_{r i}=1$

Eq. (6) shows that the characteristic resistance, $\theta_{(0)}$, can be determined from a measurement of total power and one or more measurements of temperature as a function of position on the substrate The maximum temperature is significantly reduced when the substrate power is distributed uniformly rather than concentrated in the last row. When the characteristic impedance is known, Eq. (4) can be used to find the thermal conductivity of the substrate. This analysis is shown performed for AIN and diamond substrates in the Results section below.

Effects of spreading resistance and die-attach contact resistance: In an actual module, there are two additional sources of thermal resistance on the substrate. The first is due to the resistance associated with the die-attach layer while the second is associated with heat spreading from the discrete die in the substrate. The total junction-to-ambient thermal resistance, $\theta_{j a}$, can be approximated by,

$$
\theta_{j a}=\theta_{d a}+\theta_{s p}+\theta_{1 D}+\theta_{s a}
$$

In Eq. (7), $\theta_{d a}=$ die-attach thermal resistance, $\theta_{y,}=$ spreading resistance, $\theta_{1 D}$ is the one-dimensional thermal resistance as found from Eq. (6), and $\theta_{w}=$ sink-to-ambient thermal resistance. This last quantity, $\theta_{s a}$, is dependent on the specific design of the heat sink at the $x=0$ end of the substrate.

In our experiments, we used a 0.003 in. thick film Agfilled film adhesive, to facilitate rework. With the manufacturer's reported die-attach thermal conductivity, $k_{d o}=3 \mathrm{~W} / \mathrm{m}-{ }^{\circ} \mathrm{C}$, the die-attach thermal resistance for an ATC03 die is estimated to be $\approx 0.8$ ${ }^{\circ} \mathrm{C} / \mathrm{W}$. Any interface resistance at the film-substrate or film-Si dic interfaces will increase $\theta_{d a}$ from this nominal value.

An estimate of the expected spreading resistance for the experimental geometry can be made with the analytical thermal conduction modeling code TAMS [4 ]. In TAMS, a 3D analytical solution to the heat conduction equation is found for a rectangular multilayer parallelpiped region with adiabatic side boundaries and a distribution of rectangular heat sources on the top or bottom surfaces. In addition, thermal conduction losses through attached top or bottom surface connections can be modeled in an approximate way. The work discussed in this paper was not directed to a design which minimizes $\theta_{s a}$. However,this quantity was measured and will be discussed in the next section.

\section{THERMAL TEST MODULE DESIGN AND CONSTRUCTION}

As stated earlier, the test module design was based on a 3D digital computer demonstration device shown conceptually in Fig. 1. The system design accommodates up to sixteen 2 in. by 2 in. diamond substrate MCMs cooled by direct fluid contact on both sides of one edge. A generic 2 in. wide by $1.875 \mathrm{in}$. long thermal test module was fabricated containing a $4 \times 4$ array of ATC03 test chips. The characteristics of ATC03 and its use in MCM thermal resistance studies has been discussed previously [5]. The bottom 0.375 in. was reserved for edge-cooling, leaving a 2 in. by 1.5 in. area for thin film interconnection and die placement. The basic layout for the module is shown in Fig. 3. We take $x=0$ as the upper boundary of the heat transfer region.

Each ATC03 die contains four symmetrically located polysilicon heaters capable of dissipating up to $20 \mathrm{~W}$ for a possible total of $80 \mathrm{~W}$ per die. On the substrate, thin film interconnects were made by sputtering $3 \mu \mathrm{m}$ Au over $300 \AA$ TiW and using photolithographic patterning.

A single diamond test module was fabricated using a 0.023 in. thick CVD substrate obtained from Norton Diamond Film [6]. Additional substrates wert 
constructed from $0.025 \mathrm{in}$. thick $\mathrm{Al}_{2} \mathrm{O}_{3}$ and $0.020 \mathrm{in}$. thick AIN for comparison. ATC03 die were attached using 0.003 in. thick commercial film adhesive and cured according to manufacturer's instructions.

Thermosonic bonded 0.001 in. by 0.003 in. Au ribbon was used to make die-to-substrate connections. Au plated $\mathrm{Cu}$ clips were soldered to perimeter pads on the module to provide for power input and diode thermometer output signals.

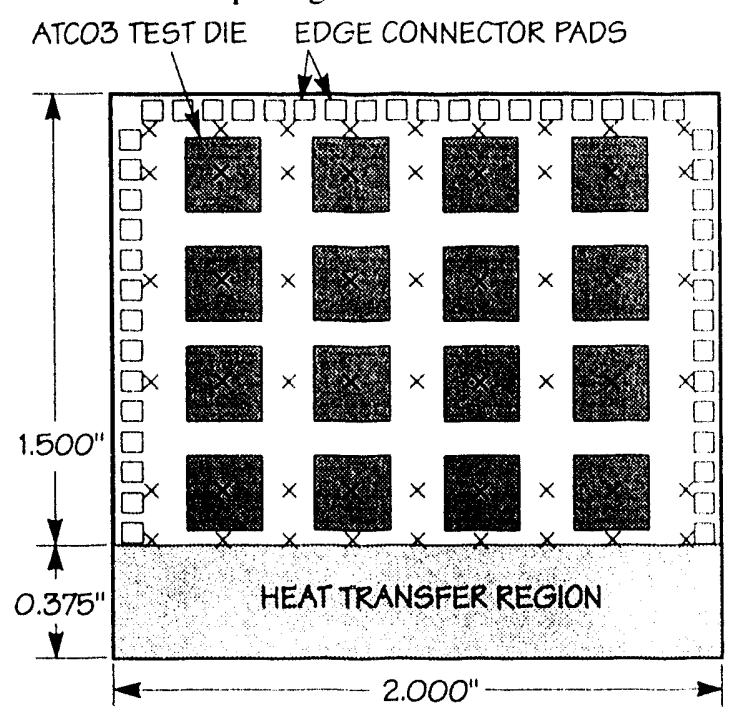

Fig. 3. Edge-cooled thermal test module containing an array of 16 ATCO3 test chips for substrate heating. The Xs show the grid used for taking fluoroptic temperature measurements.

\section{EXPERIMENTAL}

A 22-ga. stranded $\mathrm{Cu}$ wiring harness was fabricated to connect the three non-cooled edges of the module to the experimental apparatus. The thermal resistance between the top (hottest) edge to ambient through the harness was estimated to be $\theta_{e a} \approx 71{ }^{\circ} \mathrm{C} / \mathrm{W}$. This resistance is much larger than the characteristic impedance of diamond or AIN, but did affect the accuracy of measurements with the alumina substrate. Convective and radiative losses were ignored for the purposes of this experiment.

A Temptronics [6] TP350 Thermochuck was fitted with a specially made Al vise designed to uniformly clamp the module edge and provide a high conductivity thermal path between the module and the cold chuck. Edge diodes on each ATCO3 chip were calibrated between room temperature and $125^{\circ} \mathrm{C}$. The temperature coefficient at $10 \mu \mathrm{A}$ was $\partial V / \partial T \approx-1.95$ $\mathrm{mV} /{ }^{\circ} \mathrm{C}$. Besides diode thermometry, a Luxtron $|6|$ model 755 fluoroptic probe with 0.001 in. sensing head was used to measure both die surface and substrate temperatures. Diode thermometer and fluoroptic data showed close correlation for die surface temperatures. Since the latter provided both die and substrate temperature, experimental data contained in this paper are based on fluoroptic measurements.

Each substrate (diamond, AIN, alumina) was individually fitted to the Al vise and electrically connected to a patchboard using the wiring harness. For each power setting, diode thermometer forward bias voltages were measured and fluoroptic temperature readings were taken in a 6 row by 9 column grid, shown in Fig. 3.

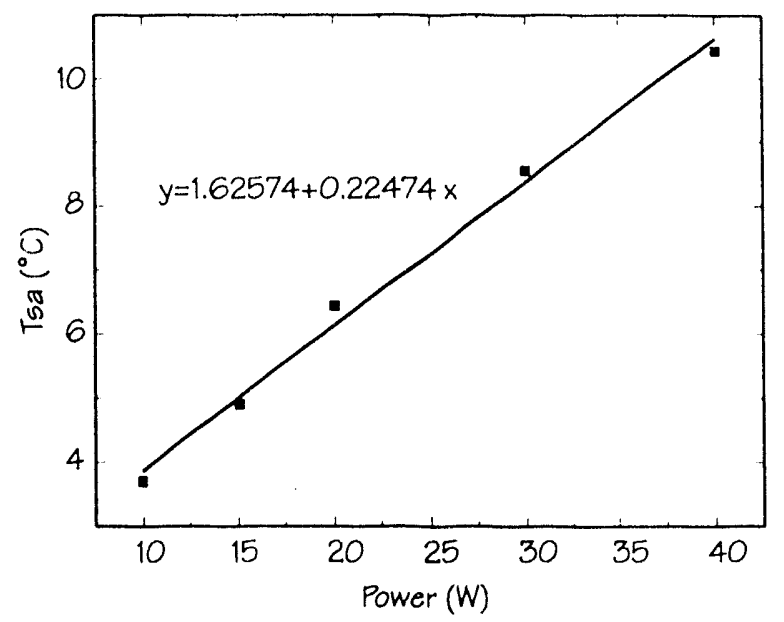

Fig. 4. Plot of $T_{s a}$ vs. power for exiraction of heatsink thermal resistance, where $T_{s a}=T(0)-T_{\text {coldchuck. }}$.

The thermal resistance of the heat sink, $\theta_{\mathrm{sa}}$, was determined by uniformly heating the top row of four ATC03 chips on the diamond module to stepped power levels from $10 \mathrm{~W}$ to $40 \mathrm{~W}$. The cold chuck temperature, $T_{\text {coldchuck }}$ was subtracted from the temperature $T(0)$ as measured at the interface between the module and the Al block. (Bottom row of Xs in Fig. 3.) The difference, $T_{s a}$, was plotted against input power in Fig. 4. A least squares fit to the data gave $\theta_{s u} \approx 0.22$ ${ }^{\circ} \mathrm{C} / \mathrm{W}$ for our experimental setup.

\section{RESULTS}

\section{ID heat transfer measurements}

To verify the adequacy of the $1 \mathrm{D}$ model for predicting temperature distributions on a heated substrate, measurements were made using two different heating configurations. In the first, only the top row of die in Fig. 3 were heated, each with the same power in order to approximate a uniformly heated strip. Substrate temperature data were obtained at a number of differen power levels with a fluoroptic probe, as described in the Experimental vection 


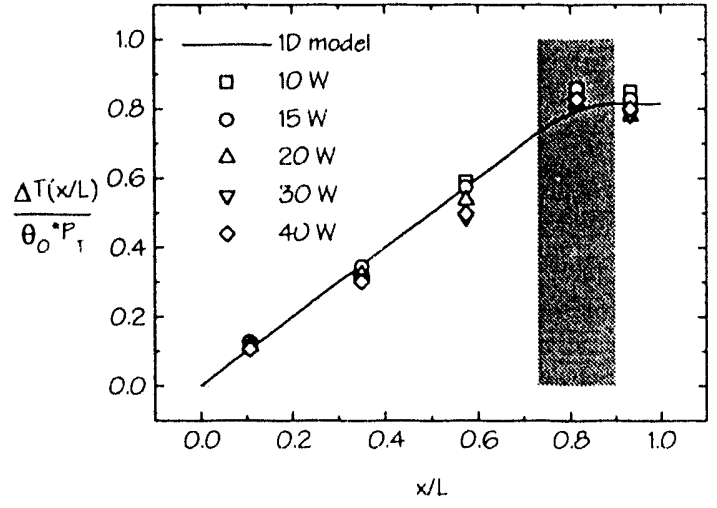

Fig. S. Diamond substrate single row heated case showing normalized measurement data superimposed on top of $I D$ model. Plot is $\Delta T(x / L) \theta_{0} * P_{T}$ vs. normalized length, $x / L$ Column marks location of heat source.

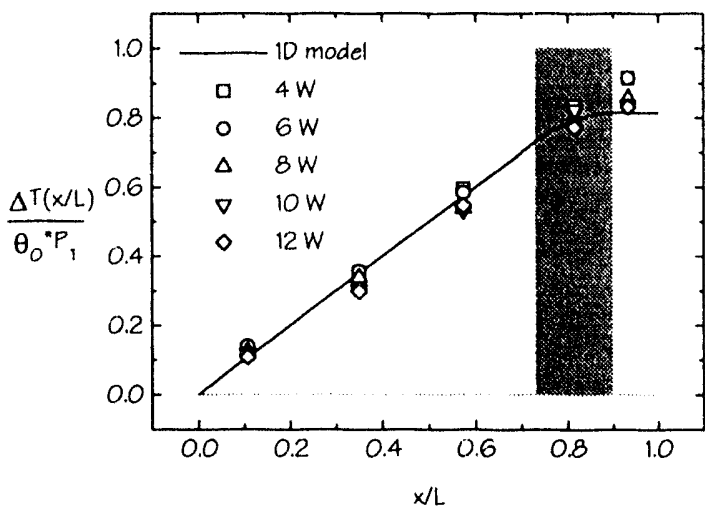

Fig. 6. AlN substrate single row heated case showing normalized measured data superimposed on top of $I D$ model. Plot is $\Delta T(x / L) \theta_{0} * P_{T}$ vs. normalized length, $x / L$. Column marks location of heat source.

Temperatures for different $y$ values were averaged to obtain an average experimental $T_{(x /)}$ function. The results of the measurements are shown in Figs. 5 and 6 for diamond and AIN substrates, respectively. In these figures, the shaded region indicates the location of the equivalent ID heated strip, which is the width of a 0.250 in. ATC 03 die. The quantity plotted on the vertical axis in these figures is the normalized temperature difference, $\Delta T(x / L) /\left(\theta_{0} P_{T}\right)$, where $\Delta T(x / L)=T(x / L)-T(0)$ is the experimental difference between the substrate temperature at $x$ and the temperature at the substrate-to-Al vise junction. The continuous curve is the prediction from Eq. (6).

For the diamond substrate case, we also made a measurement with the top three strips of die heated, with each die at the same nominal power level. The results of this measurement are shown in Fig. 7, together with the theoretical best fit curve from Eq. (6) using the least squares $\theta_{0}$, value. The characteristic thermal resistance and thermal conductivity derived from these measurements are in good agreement with the results found from the single strip heating case.

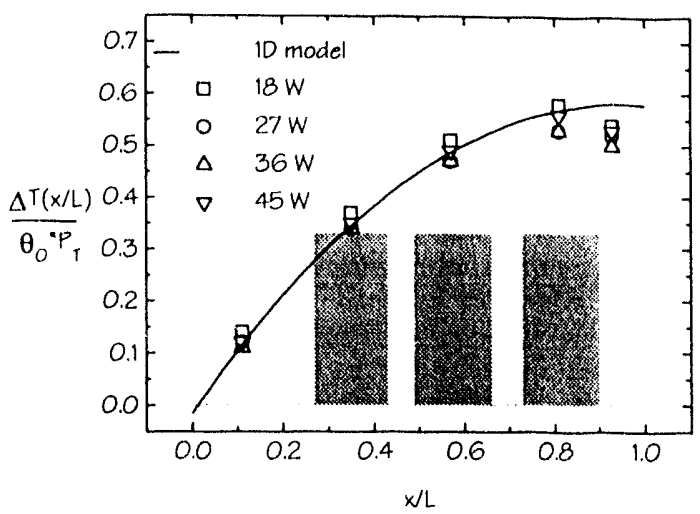

Fig. 7. Diamond substrate three top rows heated case showing measured data superimposed on top of $1 D$ model. Plot is $\Delta T(x / L) \theta_{0}{ }^{*} P_{T}$ vs. normalized length, $x / L$. Columns mark location and relative magnitudes of heat sources

The value of $\theta_{0}$ was determined by least squares fitting of the data using the solution for $\theta_{0}$ from Eq. (6). Since $\theta_{0}$ is a multiplicative factor in Eq. (6), this fitting is equivalent to averaging the solution for $\theta_{0}$ over all position measurement points and power levels. The estimated values of the thermal resistance and the standard deviation are shown in Table I. Also shown in Table $I$ is the thermal conductivity calculated from this resistance using Eq. (4). The AIN thermal conductivity is in good agreement with reported values[7].

Table I

Estimated values of thermal resistance and conductivity calculated from measured data.

\begin{tabular}{lccccc}
\hline Case & $\begin{array}{c}\theta_{0} \\
\left({ }^{\circ} \mathrm{C} / \mathrm{W}\right)\end{array}$ & $\begin{array}{c}\sigma_{\theta 0} \\
\left({ }^{\circ} \mathrm{C} / \mathrm{W}\right)\end{array}$ & $\begin{array}{c}\mathrm{k} \\
(\mathrm{W} / \mathrm{m} \cdot \mathrm{K})\end{array}$ & $\begin{array}{c}\sigma_{\mathrm{k}} \\
(\mathrm{W} / \mathrm{m} \cdot \mathrm{K})\end{array}$ & $\begin{array}{c}\mathrm{t} \\
(\mathrm{mils})\end{array}$ \\
\hline AIN - 1 strp & 5.79 & 0.40 & 248 & 24 & 20.1 \\
D - 1 strp & 0.78 & 0.07 & 1620 & 140 & 23.5 \\
D - 3 strps & 0.80 & 0.08 & 1593 & 147 & 23.5 \\
\hline
\end{tabular}

Estimates of spreading resistance from TAMS calculations using the derived thermal conductivity values were $\theta_{s p}=0.05$ and $0.33^{\circ} \mathrm{C} / \mathrm{W}$ for diamond and AIN substrates, respectively. These extimates apply to cases in which each die in a strip has the same power level. Comparison with the $\theta_{0}$ resistances in Table I 
shows that spreading resistance makes only a minor correction to total resistance in our experiment.

Although the dimensionless temperature results in Figs. 5-7 show that the 11) model can predict substrate temperatures quite accurately when the die in a given row are powered uniformly, they do not convey the significant decrease in temperature which is achieved when a diamond substrate is used in place of AIN. The actual measurements can be compared directly for the case with the top row of die uniformly heated at $10 \mathrm{~W}$ total power. In this case, the maximum substrate $\Delta T$ was $48.5^{\circ} \mathrm{C}$ for the AIN substrate and $6.6^{\circ} \mathrm{C}$ for the diamond substrate

\section{2l) heat transfer measurements}

A further comparison of substrate performance is provided in Fig. 8. In this case, the top row of ATC(O3 die was uniformly heated at $5 \mathrm{~W} /$ die for a total of 20 $W$. As seen in Fig. 8, the peak temperature measured on the diamond substrate was $T(x / L)-T(0)=\Delta T=24.3$ ${ }^{\circ} \mathrm{C}$. Due to the low spreading resistance of diamond, the substrate surrounding the heated row of die was essentially adiabatic at $\Delta T=14.8^{\circ} \mathrm{C}$. This corresponds to $\theta_{0} \approx 0.74^{\circ} \mathrm{C} / \mathrm{W}$, which is consistent with our calculations contained in Table I, and $\theta_{d a}=1.9^{\circ} \mathrm{C} / \mathrm{W}$, which is double our estimate based on the die attach adhesive film properties. This result is not unusual based on previous experiments with this adhesive and could reflect unaccounted for interface resistance and imperfect adhesion at the boundaries. We also observed an increase in die attach resistance to $\theta_{d a}>5^{\circ} \mathrm{C} / \mathrm{W}$ in subsequent measurements on all three substrates which we attribute to partial delamination of the die-toadhesive interface. This was possibly caused by steady state operation at higher than manufacturer recommended temperature. Nevertheless, it can be seen that the principle limitation in thermal performance of the diamond edge-cooled module used in this experiment is the die attach resistance. Particle loaded epoxy or Au eutectic die attach would improve both die attach resistance and high temperature operation at the expense of simple rework.

We should note that Fig. 8 shows holes in the heat transfer region of the thermal module which were designed to attach it to the 3D demonstration cube described above, and to facilitate direct fluid cooling. Experimental measurements made on the fluid cooled configuration were consistent with measurements made on the cold chuck configuration, however, due to equipment problems, only diode thermometery an the ATCO3 test chips was arablable Therefine here dit: have not been incluiled
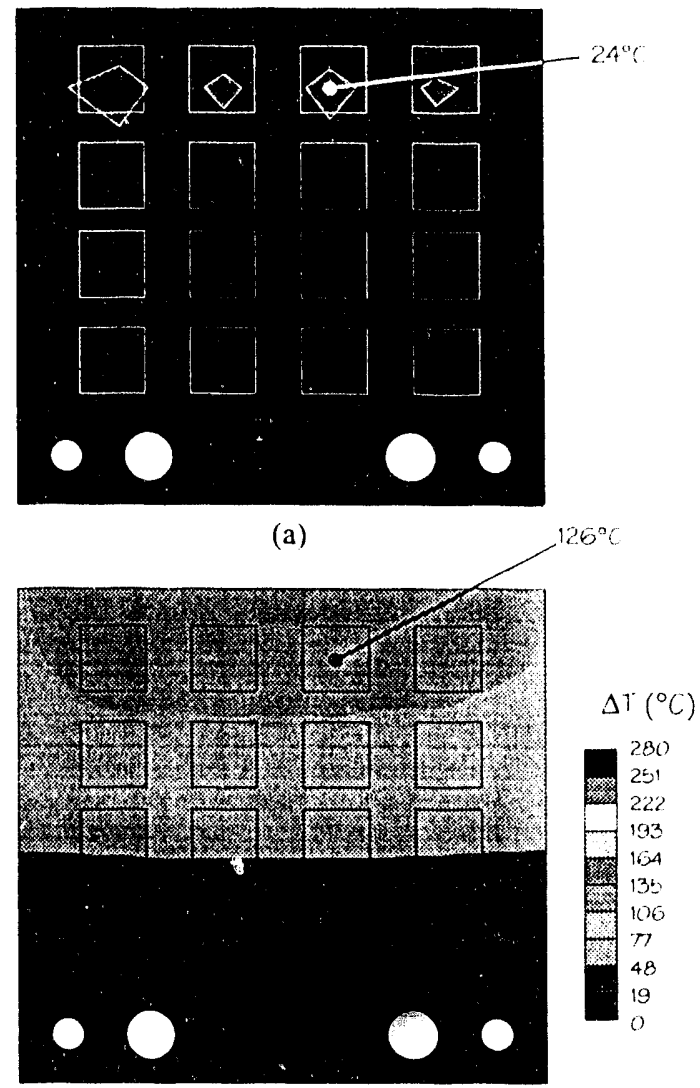

(b)

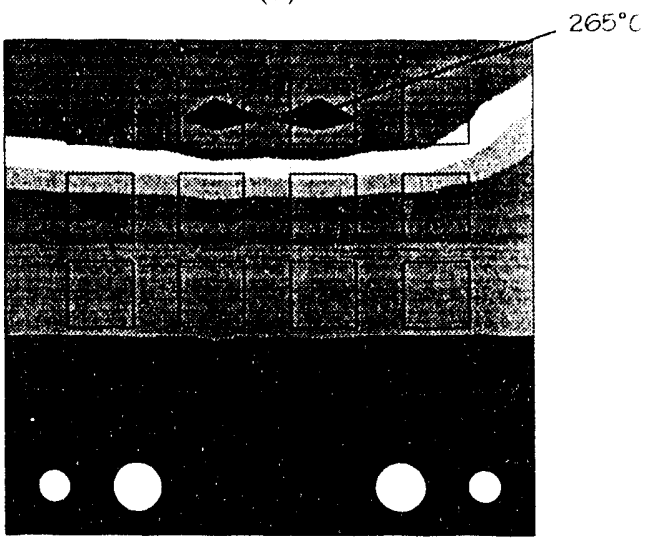

(c)

Fig. 8. Comparison of thermal performance between (a) 0.0235 in diamond, (b) 0.0201 in AIN. and (c) $0.025 \mathrm{in}$. alumina substrates while heating lop row of die with 20 W' The large diameter holes are for fluid cooling in the 31 ) demonstration cube, small holes are for sirews

\section{SUMMARY}

The measurements reported here ceprenem the ton

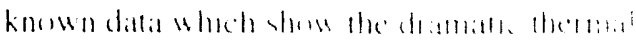

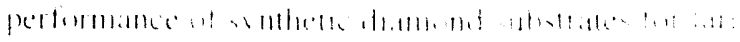

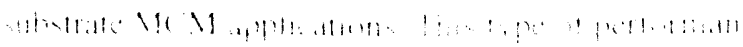

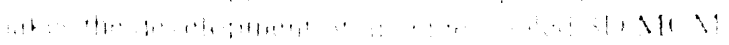


possible, at least in principle. However, to actually construct such a module, a low sink-to-ambient thermal resistance, $\theta_{\mathrm{sa}}$, must be achieved. In our experiment, $\theta_{\mathrm{sa}}$ $\approx 0.22^{\circ} \mathrm{C} / \mathrm{W}$ from Fig. 4 . The achievement of a $\theta_{\text {sa }}$ as low as this in a module such as that shown in Fig. 1 will be challanging. In addition, a method of electrically interconnecting the substrate to circuitry in the the liquid cooled chuck will have to be developed. If this can be done, the 3D MCM could have the convenience of a conventional PC card rack.

\section{REFERENCES}

[1] T. L. Moravec, G. Lu and A. Partha, "Use of CVD Diamond in High Power Packages," Proc. 1993 Int. Soc. Hybrid Manufacturing, pp. 644 649 (1993).

(2) T. J. Moravec, R. C. Eden, and D. A. Schaefer, "The Use of Diamond Substrates For Implementing 3-D MCMs," Proc. 1993 Int. Conf. \& Ex. in MCMs, pp. 86-91, April (1993).

[3] L. M. Napolitano, Jr., M. R. Daily, E. Meeks, D. Miller, D.P. Norwood, D. W. Peterson, C. A. Reber, J. E. Robles, W. Worobey, and D. Johnson, "Development of a Power Electronics Multichip Module on Synthetic Diamond Substrates," Int. J. Microcircuits \& Electronic Packaging. 16 pp. 271-278, (1993).

[4] G. N. Ellison, Thermal Computations in Electronic Equipment, Van Nostrand, NY, 1984, chaps. 7,9 .

[5] J. N. Sweet, D. W. Peterson, D. Chu, B. L. Bainbridge, R. A. Gassman, C. A. Reber, "Analysis and Measurement of Thermal Resistance in a 3-Dimensional Silicon Multichip Module Populated with Assembly Test Chips," Proc. 1993 Semiconductor Thermal Measurement and Management Sym., pp. 1-7, Feb. (1993).

[6] Reference to a particular product or company implies neither a recommendation nor an endorsement by Sandia National Laboratories, nor a lack of suitable substitutes.

[7] R. R. Tummala, E. J. Rymaszewski, Microelectronics Packaging Handbook, Van Nostrand Reinhold, NY, p. 36

* This work was performed at Sandia National Laboratories, supported by the U. S. Department of Energy under contract DE-AC04-94AL85000. 

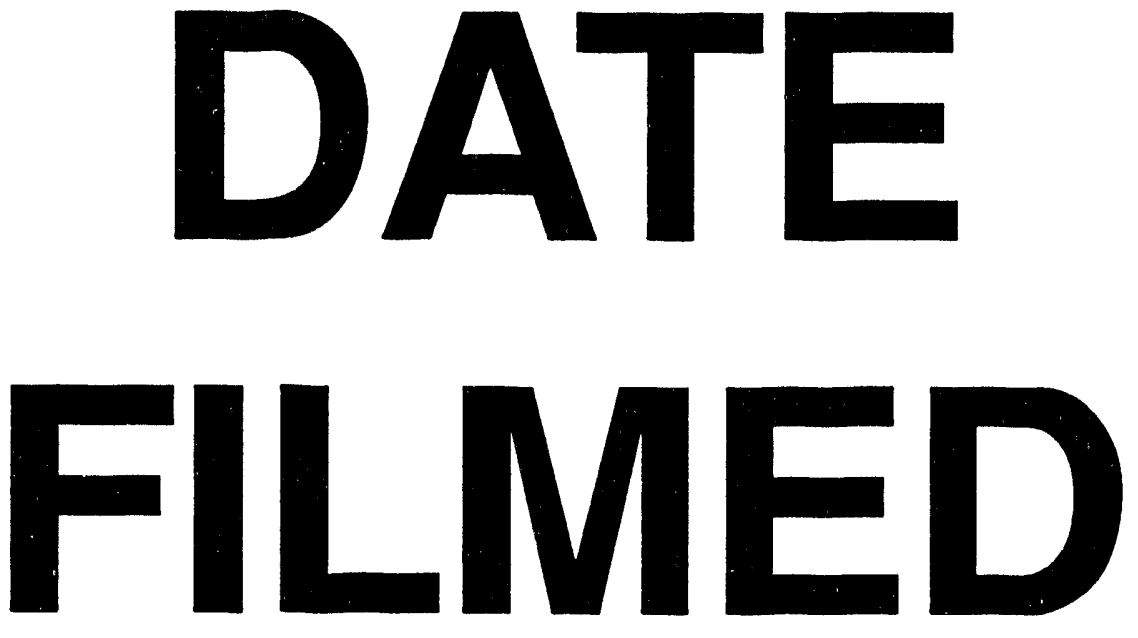

$6 / 10 / 94$
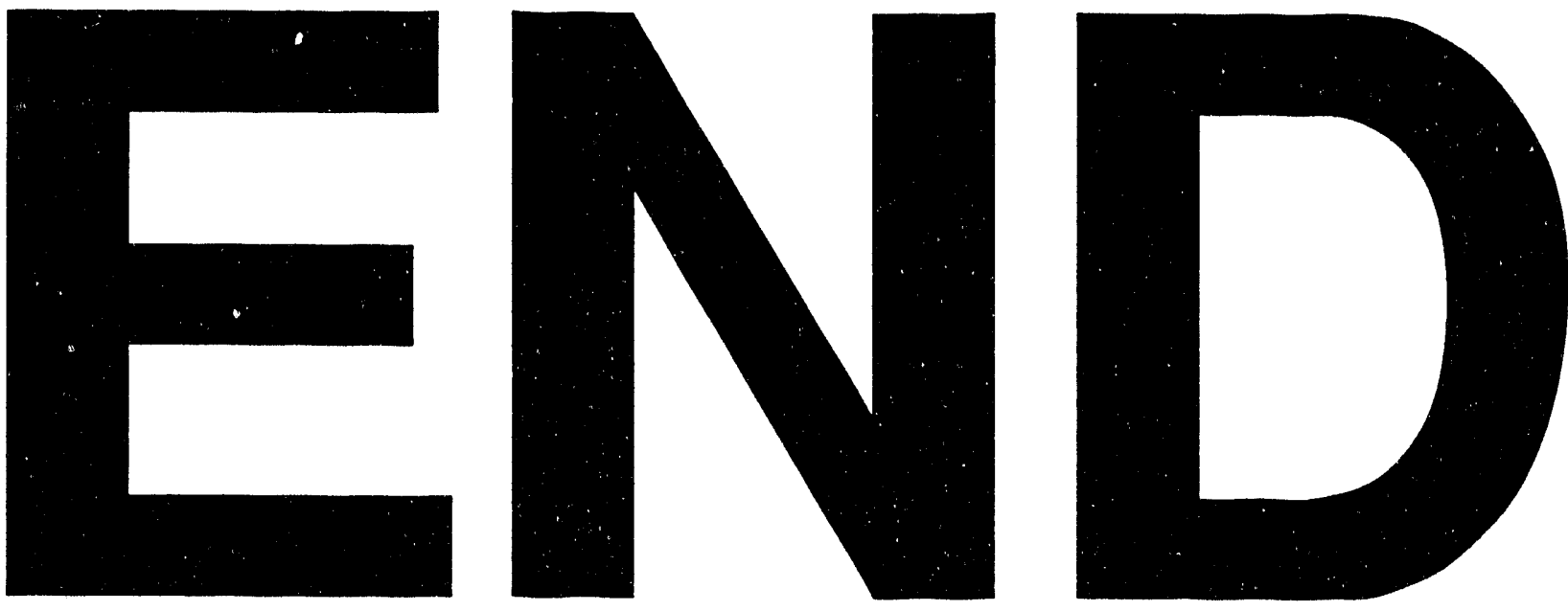
